\title{
Serum Mucin Antigen (CASA) as a Marker of Amiodarone-Induced Pulmonary Toxicity
}

\author{
Peter L. Devine ${ }^{1}$, Wendy J. Siebert ${ }^{2, \#,}$ \\ Sharon L. Morton ${ }^{3}$, Betty Scells ${ }^{4}$, \\ Rachel J. Quin ${ }^{1}$, William F. Heddle ${ }^{3}$, \\ Paul V. Zimmerman ${ }^{4}$, and Peter J. Donohoe ${ }^{5}$ \\ ${ }^{1}$ Department of Obstetrics \& Gynaecology, \\ University of Queensland, Brisbane, Australia \\ ${ }^{2}$ Department of Pharmacy, Flinders Medical \\ Centre, Adelaide, Australia \\ ${ }^{3}$ Department of Medicine, Flinders Medical \\ Centre, Adelaide, Australia \\ ${ }^{4}$ Department of Thoracic Medicine, Prince \\ Charles Hospital, Chermside, Queensland, \\ Australia \\ ${ }^{5}$ Department of Immunology, Allergy and \\ Arthritis, Flinders Medical Centre, Adelaide, \\ Australia
}

\begin{abstract}
Amiodarone is used to treat lifethreatening cardiac arrhythmias. Amiodarone-induced pulmonary toxicity (APT) can be difficult to diagnose. APT may result in increased mucus production and mucin expression. Thus, serum mucin-1 was evaluated as a marker for amiodarone-induced pulmonary toxicity. Concentrations of mucin-1 in peripheral blood were determined using cancer-associated serum antigen (CASA) assay in patients taking amiodarone. Eight of ten patients who developed major amiodarone toxicity had high serum CASA levels. Patients with toxicity had a significantly higher mean rank CASA concentration compared with those without major toxicity. CASA shows potential as a marker for amiodarone-induced toxicity, particularly pulmonary toxicity.
\end{abstract}

KEYWORDS: mucin, CASA, amiodarone, pulmonary toxicity

\footnotetext{
\# Correspondence: Wendy J. Siebert, Flinders Medical Centre, Bedford Park, S. Australia, 5042, Tel.: + 6188204 5511, Fax: +61 882045841 .
}

\section{INTRODUCTION}

Amiodarone hydrochloride, a class III antiarrhythmic drug, is used to treat life-threatening supraventricular and ventricular tachyarrhythmias; in particular, arrhythmias resistant to other agents [17]. Desethylamiodarone is its major active metabolite [9]. Amiodarone has a wide profile of adverse effects involving a number of organ systems [17]. Amiodarone-induced pulmonary toxicity, the most serious non-cardiac side effect, has been reported to occur in $2-13 \%$ of patients $[2,15]$, with up to $30 \%$ mortality [8]. Treatment of APT generally requires withdrawal of the drug and alternative treatment is currently limited [17].

Early recognition of APT is difficult as it relies upon non-specific symptoms, signs, radiological changes and abnormalities on routine pulmonary function testing [11]. Radiographic features of APT are characteristic but non-specific. A decrease in carbon monoxide lung diffusing capacity (DLCO) is a sensitive indicator of APT in the presence of symptoms and radiological findings, however a reduction in DLCO in asymptomatic patients taking amiodarone does not indicate pulmonary toxicity [13]. Patients taking amiodarone for ventricular arrhythmias often have concomitant conditions, such as congestive cardiac failure (CCF), chronic obstructive airways disease (COAD), infection or malignancy that need to be excluded as the cause of clinical findings. Furthermore, unusual clinical manifestations of APT have been reported [14]. However, the main diagnostic difficulty occurs when the non-specific symptoms of APT are masked by the symptoms of overt cardiac failure in a critically ill patient, with the diagnosis of APT only occurring after treatment of underlying cardiac disease [11]. This delay in establishing the diagnosis of APT may 
increase the risk of a fatal outcome [11]. Due to increasing use of amiodarone [18], pulmonary toxicity is likely to occur more frequently. Thus, a test that could predict the clinical occurrence of APT and possibly differentiate it from cardiac failure would be extremely useful.

Evidence suggests that APT is due to direct toxic effects of the drug or immunological mechanisms [19]. Amiodarone pulmonary toxicity is associated with hyperplasia of respiratory epithelium, thickening and oedema of the pulmonary interstitium and variable degrees of inflammation involving neutrophilic and mononuclear cells [8].

Mucus hypersecretion is a characteristic feature of several human airways diseases [10]. Mucin glycoproteins (mucins) are the major components of mucus, the mucosal layer that protects and lubricates pulmonary epithelium $[3,20]$. APT may result in mucin hypersecretion in an attempt to protect the lung epithelium. Assays to detect high molecular weight glycoproteins of the mucin group are available. One such assay is the CancerAssociated Serum Antigen (CASA) assay, an ELISA, that uses monoclonal antibodies (mabs) to detect a peptide epitope on the MUC1 mucin [6]. This has been reported to be elevated in the serum of patients with malignant lung disease [4,7].

Our hypothesis is that amiodarone induced pulmonary toxicity is associated with mucin hypersecretion. This will be detectable by the CASA assay and thus aid diagnosis of APT. The aims of this study were: 1) to ascertain whether CASA levels were elevated in APT; and 2) to compare CASA levels in APT and other respiratory diseases.

\section{METHODS}

\section{Patients}

Subjects included: 47 patients enrolled in a prospective follow-up study of amiodarone at Flinders Medical Centre; and 157 patients with benign respiratory disease and 258 with lung cancer attending clinics at The Prince Charles Hospital, Brisbane, as well as 54 randomly selected healthy blood donors. Amiodarone study patients had consented to prospective monitoring and blood sampling at the time of commencing amiodarone; the age, gender, smoking history, duration of amiodarone therapy, dose, annual chest x-ray findings, six monthly pulmonary function tests (including diffusing capacity), and serial serum biochemistry, plasma amiodarone and desethylamiodarone concentrations, adverse effects including toxicity and clinical findings were documented. Patients with at least three serial samples were included in this study. Blood was collected by venipuncture. The sample was centrifuged and the serum or plasma collected, aliquotted, and stored at $-70^{\circ} \mathrm{C}$ until assay. Serum antigen (CASA) levels were determined retrospectively.

\section{Assay}

The CASA assay is a dual determinant assay that incorporates two anti-MUC1 core protein monoclonal antibodies (BC2 and $\mathrm{BC} 3$ ) [5]. CASA concentrations were determined in serum and plasma samples. Only small differences between values obtained with sera or plasma have been reported using this assay. All samples were assayed in duplicate and determined by interpolation against a standard curve (2-64 units $/ \mathrm{mL}$ ). Positive and negative controls were included in all assays. The cutpoint of $9 \mathrm{U} / \mathrm{mL}$ for CASA provided the best differentiation between patients with and without amiodarone toxicity.

\section{Diagnostic Criteria}

The criteria for diagnosis of amiodarone pulmonary toxicity, adapted from Magro et. al. [13] included: 1) recent onset dyspnea and/or persistent dry cough; and/or 2) new or progressive infiltrates on chest X-ray; and 3) diffusing capacity (DLCO) decreasing by $\geq 20 \%$ or DLCO $\leq 60 \%$ of predicted; and 4) improvement on cessation of amiodarone. The diagnosis was also dependent on the exclusion of other causes, such as congestive cardiac failure, infection, chronic airflow obstruction and 
alternative causes of pulmonary fibrosis.

If the diagnosis of amiodarone pulmonary toxicity was suspected, amiodarone was ceased. The final diagnoses based on ADEC classifications [1] were as follows: patients who met the diagnostic criteria were classified as probable amiodarone pulmonary toxicity if drug withdrawal resulted in resolution of symptoms, and possible amiodarone pulmonary toxicity if there was only partial recovery on cessation of amiodarone.

\section{CASA Levels Correlation with Cardiac Function, Pulmonary Gas Transfer and Plasma Drug Concentrations}

First, cardiac function was considered. Patients taking amiodarone frequently have poor left ventricular function; thus the differential diagnosis of APT and congestive cardiac failure may occur concomitantly. Therefore, we examined the relationship between mucin production as measured by the CASA assay and the presence of cardiac failure (using the New York Heart Association functional criteria).
Second, the diffusing capacity of carbon monoxide (DLCO) is a useful aid in diagnosing and monitoring APT particularly after drug cessation in most patients. Thus, we examined the relationship between mucin production, as measured by the CASA assay and the diffusing capacity of carbon monoxide corrected for alveolar volume $(\mathrm{KCOc})$. In addition, we also examined whether CASA was related to trough plasma amiodarone and desethylamiodarone concentrations.

\section{RESULTS}

\section{CASA Levels in Different Diagnostic Groups}

Plasma or serum from 516 patients was examined. The distribution of CASA in the different diagnostic patient groups is shown (Figure 1, Table 1). A cutpoint of 9 units $/ \mathrm{mL}$ (greater than twice the upper limit of the control population) provided the greatest sensitivity $(80 \%)$ for predicting toxicity in patients taking

Table 1

Number of people in the amiodarone group and control group with elevated CASA concentrations $(\geq 9$ Units $/ \mathrm{mL}$ )

\begin{tabular}{lcc}
\hline Group & $\begin{array}{l}\text { number with elevated CASA/ } \\
\text { total number in each subgroup (\%) }\end{array}$ \\
\hline Amiodarone group & & $(0 \%)$ \\
\hline $\begin{array}{l}\text { No serious toxicity } \\
\text { Lung toxicity } \\
\text { probable }\end{array}$ & $0 / 37$ & $(75 \%)$ \\
$\quad$ possible, confirmed & $3 / 4$ & $(100 \%)$ \\
Other toxicity & $3 / 4$ & \\
$\quad$ hepatotoxicity & $1 / 1$ & $(0 \%)$ \\
$\quad$ thyroiditis & $1 / 1$ & $(6 \%)$ \\
\hline Control group & & \\
\hline Healthy donors & $0 / 54$ & \\
Benign lung disease & $10 / 157$ & \\
$\quad$ obstructive disease & $3 / 53$ & \\
$\quad$ inflammatory disease & $2 / 21$ & \\
$\quad$ infection & $5 / 38$ & \\
$\quad$ other & $0 / 95$ & \\
Lung Cancer & $60 / 258$ & \\
\# including two patients, initially diagnosed with APT, whose subsequent follow-up \\
supported a final diagnosis of cardiac failure.
\end{tabular}




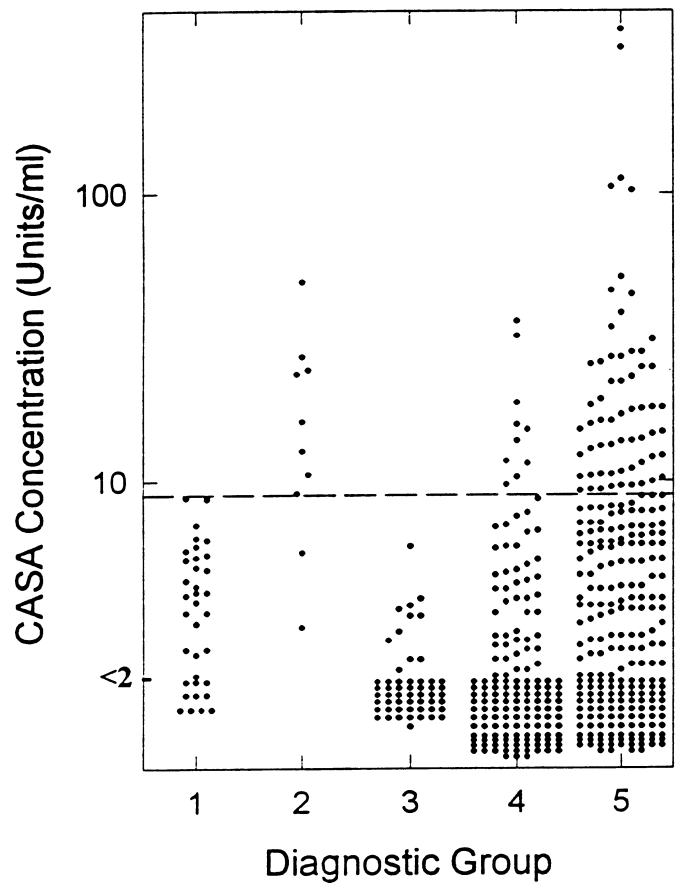

Fig. 1. The level of CASA in the Amiodarone and control groups. Groups shown are: (1) patients taking amiodarone without toxicity; (2) patients with amiodarone toxicity; (3) healthy blood donors; (4) patients with benign respiratory conditions; (5) patients with lung cancer. A CASA level of 9 units $/ \mathrm{mL}$, the proposed cutpoint, is represented by a broken line.

amiodarone. Of eight patients diagnosed with APT, the CASA level was elevated in six cases (range: 9-49 units/ml). In two patients with other amiodarone-induced toxicity (one hepatotoxicity, one thyroiditis) the CASA levels were elevated (13 and 16 units $/ \mathrm{mL}$ respectively). Thirty-five patients taking amiodarone without these serious toxicities, (and two patients thought initially to have APT, but subsequently diagnosed with congestive heart failure,) did not have elevated CASA levels. In addition, no healthy control patients $(0 / 54)$ showed elevation of CASA (range $<2.0-5.9$ units $/ \mathrm{mL})$. Six percent $(10 / 157)$ of patients with non-malignant respiratory disease showed CASA $\geq 9.0$ units $/ \mathrm{mL}$; thirteen percent $(5 / 38)$ of whom had infections, which are relatively easy to diagnose. Furthermore, twenty three percent of patients $(60 / 258)$ with lung cancer also showed elevated CASA, with elevation in all stages and histological types of disease.

\section{Amiodarone Patient Characteristics}

The characteristics of the total amiodarone patient population $(n=47)$ : consisting of 37 patients taking amiodarone without toxicity and ten patients with amiodarone-induced toxicity are shown in Table 2. The total amiodarone patient group consisted of eight females and thirty-nine males, however amiodarone toxicity was more common in males than females $(23 \%$ compared with $13 \%$ respectively). The average age was similar for patients with and without toxicity. The mean dose of amiodarone for patients without toxicity $(290 \mathrm{mg} / \mathrm{day})$, and with toxicity (300 mg/day), were comparable to the mean dose of amiodarone for all patients ( $300 \mathrm{mg} /$ day). The duration of amiodarone therapy was shorter in patients with toxicity ( 60 weeks) compared with the total group (average 79 weeks) as a consequence of amiodarone cessation when toxicity was diagnosed. 
Table 2

Characteristics for all patients taking amiodarone, with and without toxicity

\begin{tabular}{cccc}
\hline & All amiodarone patients & No amiodarone toxicity & Amiodarone toxicity \\
\hline $\mathrm{n}$ & 47 & 37 & 10 \\
\hline Gender & 8 & 7 & 1 \\
$\mathrm{~F}$ & 39 & 30 & 9 \\
$\mathrm{M}$ & 65 & 64 & 67 \\
$\begin{array}{c}\text { Age (years) } \\
\text { mean }\end{array}$ & $21-82$ & $21-82$ & $53-74$ \\
range & & & 300 \\
$\begin{array}{l}\text { Dose (mg/day) } \\
\text { mean }\end{array}$ & 290 & 290 & $150-400$ \\
range & $100-400$ & $100-400$ & 60 \\
$\begin{array}{l}\text { Duration (weeks) } \\
\text { mean }\end{array}$ & 79 & 84 & $12-156$ \\
range & $12-182$ & $12-182$ & \\
\hline
\end{tabular}

\section{Use of CASA in Monitoring Patients with Suspected APT}

Of ten patients with an initial diagnosis of suspected APT, four were classified as probable APT and six as possible APT. Three of four patients with probable APT, and three of six patients with possible APT, had an elevated CASA above 9 units $/ \mathrm{ml}$. The diagnosis of APT was difficult in the latter group because of concomitant disease.

Of the six patients with possible APT, two patients with both APT and chronic obstructive airways disease had an elevated CASA. One patient with symptoms of APT had their amiodarone ceased, resulting in clinical improvement and blood taken at their next clinic visit revealed a slightly elevated CASA level. One patient with possible APT and concomitant COAD and CCF did not have an elevated CASA. In the other two patients with possible APT, clinical progress confirmed a diagnosis other than APT: their final diagnosis was severe congestive cardiac failure and they did not have an elevated CASA.

In all cases of suspected APT, treatment involved ceasing amiodarone therapy. Steroids were not routinely used. In all patients with elevated CASA, cessation of amiodarone resulted in resolution or improvement of symptoms and a fall in CASA to below 9 units $/ \mathrm{mL}$.
Two patients, with other amiodarone-induced toxicities, had an elevated CASA. One patient developed amiodarone hepatotoxicity. Cessation of amiodarone resulted in liver function tests and CASA levels returning to within the normal range. At the time of diagnosis of amiodaroneinduced thyroiditis, another patient had an elevated CASA; both the thyroiditis and elevated CASA resolved with time.

In summary, three of four patients with probable amiodarone pulmonary toxicity and three patients with possible amiodarone toxicity had an elevated CASA. In the latter group with possible APT, two patients also had COAD. One patient with concomitant $\mathrm{COAD}$ and $\mathrm{CCF}$, and two patients whose subsequent diagnosis was heart failure, did not have elevated CASA.

Therefore, CASA levels, may assist in distinguishing between amiodarone pulmonary toxicity and severe congestive cardiac failure in some patients. In addition, two patients with other amiodarone toxicities (hepatotoxicity and thyroiditis) had elevated CASA.

Due to the skewness of CASA concentration distributions, a Mann - Whitney U Test was used to compare CASA concentration in patients with and without toxicity. Patients with toxicity had a median CASA concentration of 14.4 units $/ \mathrm{mL}$ compared with those without toxicity who had a median of 4.0 units $/ \mathrm{mL}$. The results of the Mann-Whitney U Test showed that patients 


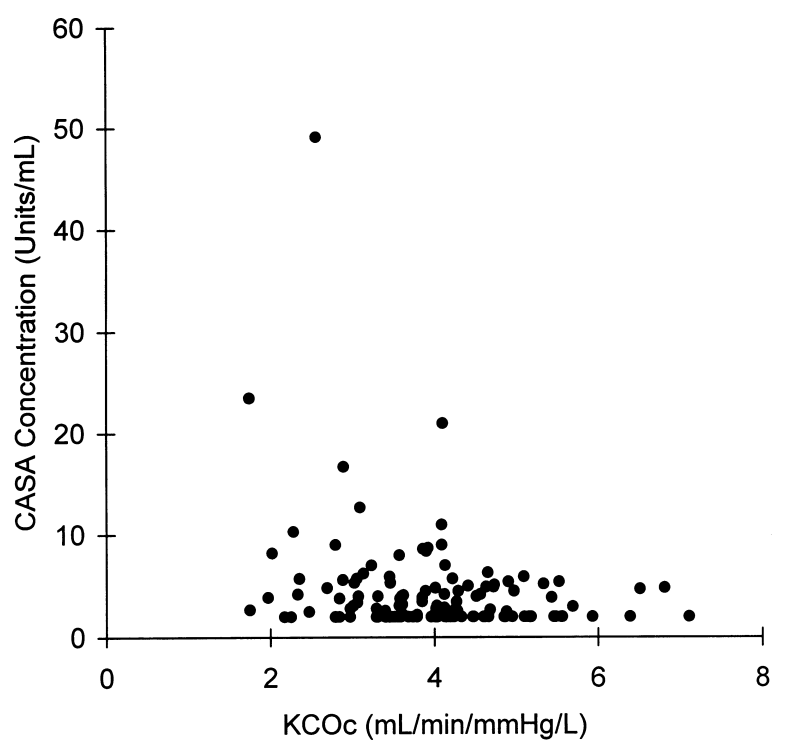

Fig. 2. A scatter plot of CASA levels and KCOc (lung diffusing capacity) measurements in all patients taking amiodarone.

with toxicity had significantly higher mean rank CASA concentration $(\mathrm{p}<0.0001)$.

CASA levels were compared with degree of congestive cardiac failure, lung diffusing capacity for carbon monoxide and plasma amiodarone and desethylamiodarone concentrations. There was no correlation between CASA levels and CCF status, using NYHA functional class criteria; nor was there a correlation between CASA and amiodarone and desethylamiodarone plasma concentrations. A weak negative correlation between CASA and KCOc (Figure 2) was demonstrated. Only six percent of the variation in CASA was accounted for variation in KCOc.

\section{DISCUSSION}

Amiodarone is very useful treatment for refractory arrhythmias [17]. However, adverse effects of amiodarone, particularly pulmonary toxicity, may limit its clinical use [2]. At present, clinical review, respiratory function testing and radiographic studies are performed to monitor for APT [16]. Because amiodarone is used for the treatment of life-threatening arrhythmias, the accurate diagnosis of pulmonary toxicity is desirable before cessation of the drug. A simple reliable test to screen for and assist in the diagnosis of amiodarone pulmonary toxicity is needed.

An elevated Cancer-Associated Serum Antigen (CASA) was shown in this study to be associated with amiodarone toxicity, particularly pulmonary toxicity. Five patients with probable amiodarone related toxicity ( 3 lung, 1 hepatic, 1 thyroiditis) and three patients with possible pulmonary toxicity, showed elevation of this serum marker ( $\geq 9$ units $/ \mathrm{mL}$ ). CASA appeared to be useful in distinguishing between APT and CCF in two patients. CASA was elevated in only $6 \%$ of patients with benign respiratory disease, the majority of whom had acute infections that are relatively easily to diagnose. No healthy controls had elevated CASA.

An elevated CASA has been reported in the serum of patients with lung cancers [4,7]. It has been proposed that overproduction of the protein core of the MUC1 mucin in cancer cells leads to underglycosylation and the exposure of novel epitopes detected in the CASA assay [6]. Due to the high expression of CASA in the serum of patients with amiodarone toxicity it is likely that 
the drug also leads to overexpression of respiratory MUC1. This is particularly interesting in light of the proposed role of mucin in the protection of the epithelial surface from foreign agents [3,20], and may assist in elucidating the etiology of APT.

These findings suggest that CASA may be useful as an adjunct in the diagnosis of amiodarone toxicity, particularly the difficult diagnosis of amiodarone pulmonary toxicity. It also may assist in differentiating APT and CCF in some patients. Further investigation is desirable for two reasons: first, to increase the knowledge regarding the etiology of amiodarone toxicity; and second, to further investigate the usefulness of CASA as a potential serum marker in the diagnosis and management of patients with suspected amiodarone toxicity.

\section{References}

[1] Australian Drug Evaluation Committee, Report of adverse drug reactions, No.5. Australian Government Publishing Service (1981), Canberra.

[2] Counihan, P.J. and McKenna, W.J. Risk benefit assessment of amiodarone in the treatment of cardiac arrhythmias. Drug Safety 5, (1990) 286304.

[3] Devine, P.L. and McKenzie, I.F.C. Mucins: structure, function, and associations with malignancy. Bioessays 14, (1992) 619-625.

[4] Devine, P.L., McGuckin, M.A., Ramm, L.E., Pee, D., Long, S. and Ward, B.G. Serum mucin antigens CASA and MSA in benign and malignant tumours of the breast, ovary, lung, pancreas, bladder, colon, and prostate: a blind trial with 420 patients. Cancer 72, (1993) 2007-2015.

[5] Devine, P.L. and Quin, R.J. Use of plasma in serum tumor marker assays CASA and MSA. Eur. J. Clin. Chem. Clin. Biochem. 32, (1994) 629-630.

[6] Devine, P.L., Warren, J.A., Ward, B.G., McKenzie, I.F.C. and Layton, G.T. Glycosylation and the exposure of tumor-associated epitopes on mucins. J. Tumor Marker Oncol. 5, (1990) 11-26.

[7] Devine, P.L., Yarker, J., Fong, K., McGuckin, M.A., Thynne, G.S. and Zimmerman, P.V. Tumour markers CYFRA 21-1, TPA, TPS, NSE, CEA, CASA and MSA in malignant and benign lung disease. Int. J. Oncol. 4, (1994) 1129-1135.

[8] Dunn, M. and Glassroth, J. Pulmonary complications of amiodarone toxicity. Prog. Cardiovascular Dis. 31, (1989) 447-453.

[9] Falik, R., Flores, B.T., Shaw L., Gibson, G.A., Josephson, M.E. and Marchlinski, F.E. Relationship of steady-state serum concentrations of amiodarone and desethylamiodarone to therapeutic efficacy and adverse effects. Am. J. Med. 82, (1987) 1102-1108.

[10] Jany, B. and Basbaum, C.B. Mucin in disease. Modification of mucin gene expression in airways disease. Am. Rev. Respir. Dis. 144, (1991) S38S41.

[11] Jessurun, G.A.J., Boersma, W.G. and Crijns, H.J.G.M. Amiodarone-induced pulmonary toxicity. Predisposing factors, clinical symptoms and treatment. Drug Safety 18, (1998) 339-344.

[12] Kennedy, J.I. Clinical aspects of amiodarone pulmonary toxicity. Clin. Chest Med. 11, (1990) 119-129.

[13] Magro, S.A., Lawrence, E.C. and Wheeler, S.H. Amiodarone pulmonary toxicity: prospective evaluation of serial pulmonary function tests. $J$. Am. Coll. Cardiol. 12, (1988) 781-788.

[14] McNeil, K.D., Firouz-Abadi, A., Oliver, W. and Zimmerman, P.V. Amiodarone pulmonary toxicity - three unusual manifestations. Aust. N.Z. J. Med. 22, (1992) 14-18.

[15] Naccarelli, G.V., Rinkenberger, R.L., Dougherty, A.H. and Fitzgerald, D.M. Adverse effects of amiodarone, pathogenesis, incidence and management. Med. Toxicol. Adverse Drug Experience 4, (1989) 246-253.

[16] Nicolet-Chatelain, G., Prevost, M.C., Escamilla, R. and Migueres, J. Amiodarone-induced pulmonary toxicity. Immunoallergologic tests and bronchoalveolar lavage phospholipid content. Chest 99, (1991) 363-369.

[17] Podrid, P.J. Amiodarone. Reevaluation of an old drug. Annals Intern. Med. 122, (1995) 689-700.

[18] Pollak, P.T. Amiodarone: Historical overview and development. Pharmacotherapy 18, (1998) 121S$126 \mathrm{~S}$.

[19] Reasor, M.J. and Kacew, S. An evaluation of possible mechanisms underlying amiodaroneinduced pulmonary toxicity. Proc. Soc. Expt. Biol. Med. 212, (1996) 297-304.

[20] Rose, M.C. Mucins: structure, function, and role in pulmonary disease. Am. J. Physiol. 263, (1992) L413-L429. 


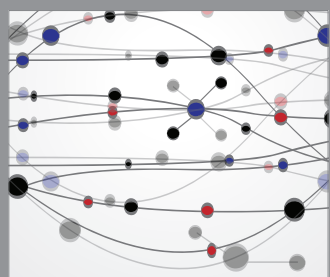

The Scientific World Journal
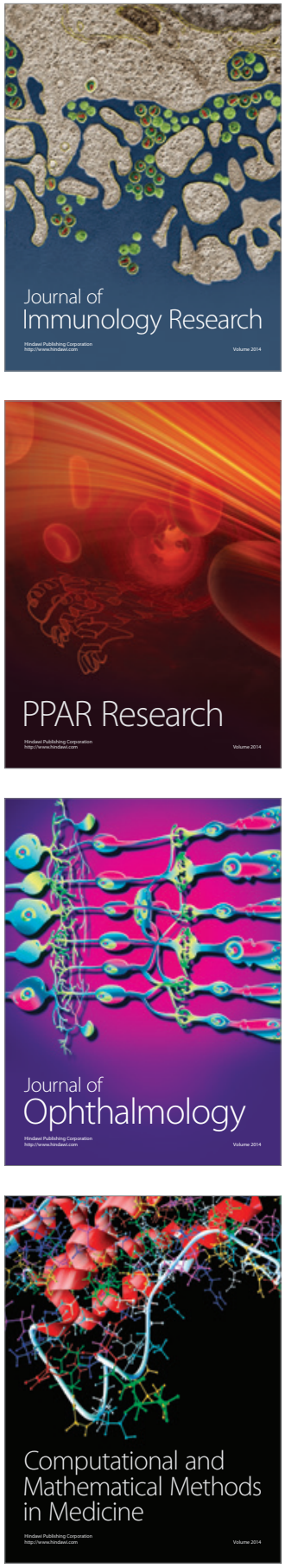

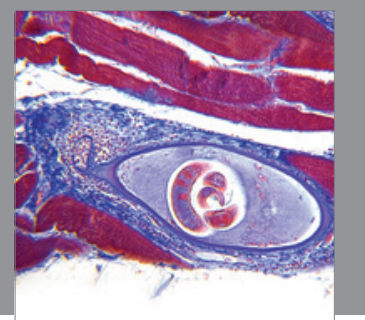

Gastroenterology

Research and Practice
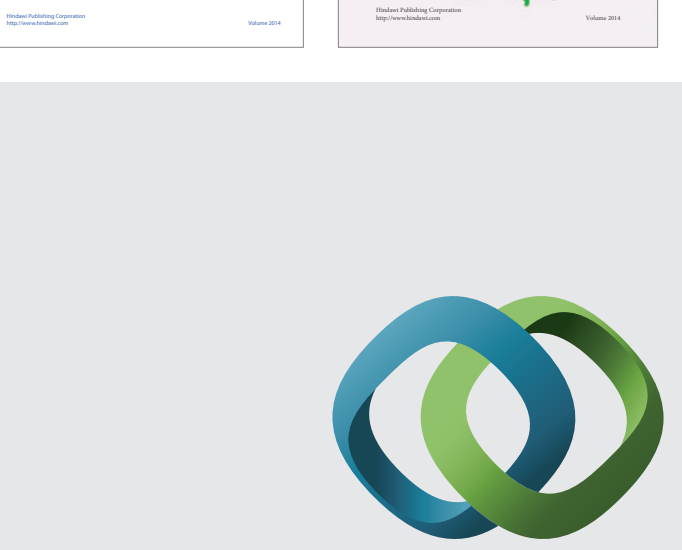

\section{Hindawi}

Submit your manuscripts at

http://www.hindawi.com
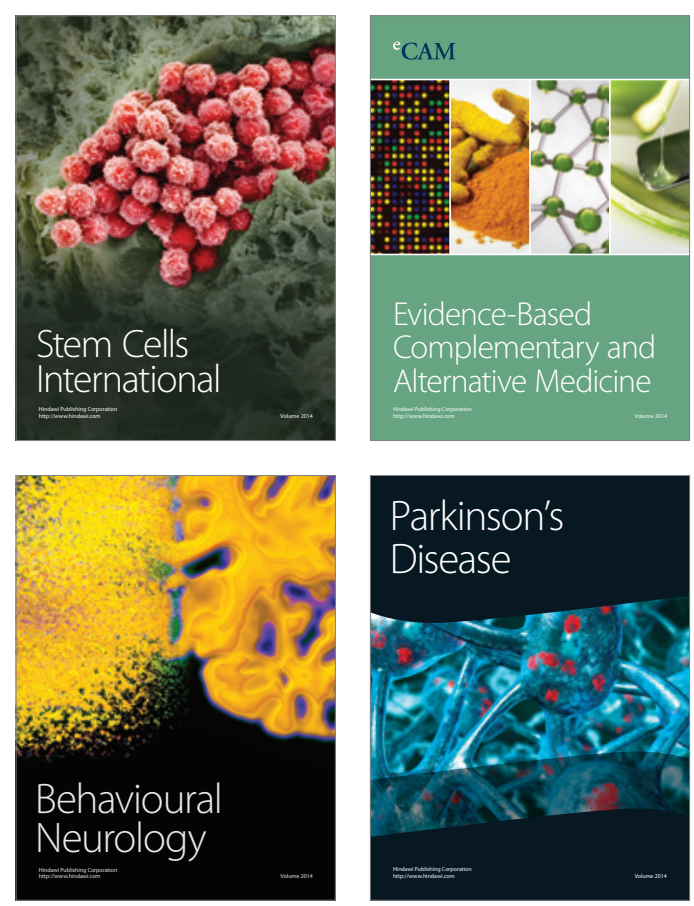

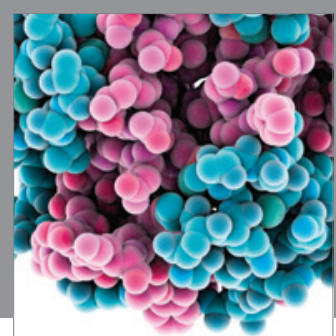

Journal of
Diabetes Research

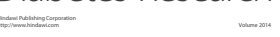

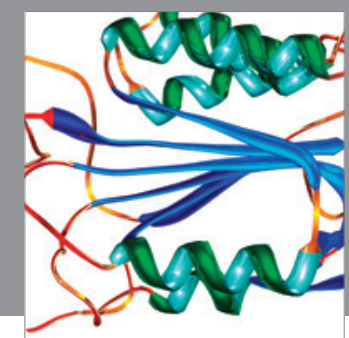

Disease Markers
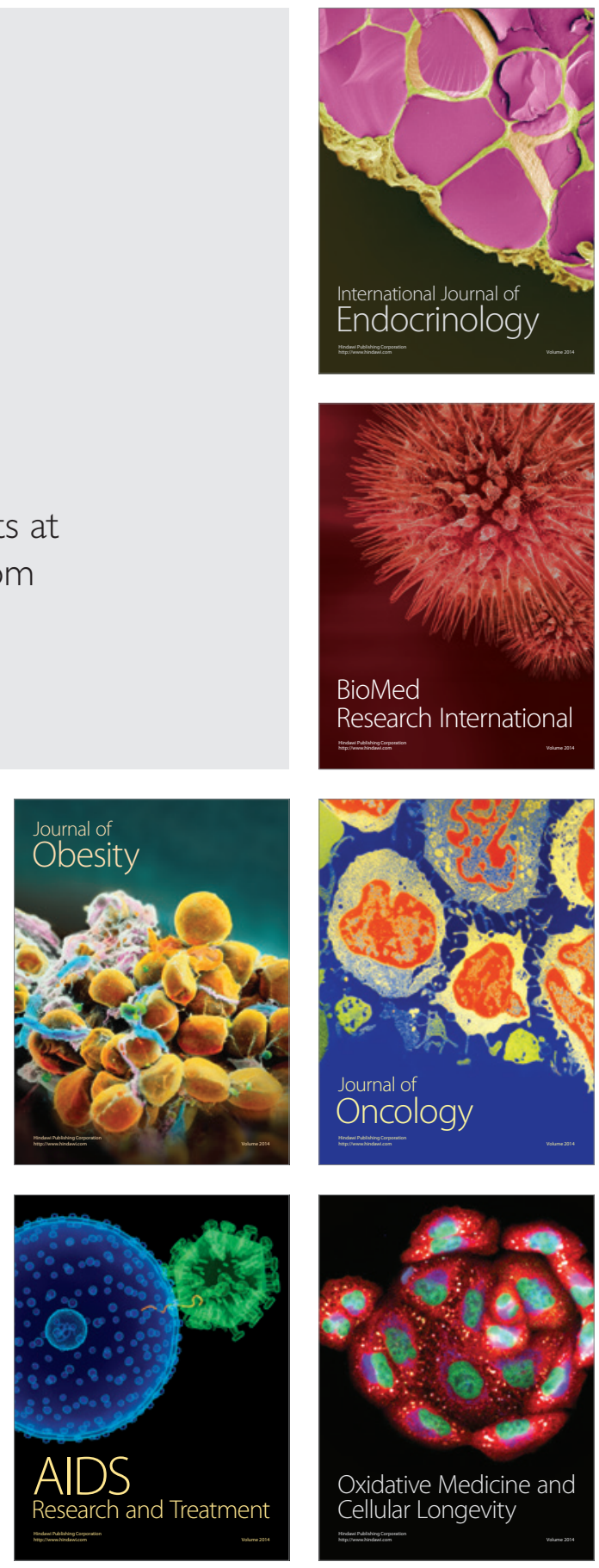